\title{
The prevalence of gastroesophageal reflux disease and reflux esophagitis in dialysis patients: between facts and fiction
}

\author{
Ibrahiem Saeed Abdul-Rahman
}

Department of Internal Medicine, Nephrology Division, King Fahd University Hospital, Dammam University, Saudi Arabia

Correspondence: Ibrahiem Saeed Abdul-Rahman, Department of Internal Medicine, Nephrology Division, King Fahd Hospital of the University, Dammam University, PO Box 40032, Al-Khobar, 31952, Saudi Arabia.

Tel. +966.3.8966697 - Fax: +966.3.8966741

E-mail: isaeed.kfu.edu@gmail.com ; isaeed@ud.edu.sa

Key words: gastroesophageal reflux disease, esophagitis, hemodialysis, peritoneal dialysis, diabetes mellitus, body mass index, H. pylori.

Contributions: ISA-R organized the patient's records, coordinated with the endoscopy unit, followed-up the pathology results, carried out the statistical tests and wrote the manuscript. A-AA-Q performed the endoscopy procedures, shared in the study design and reviewed the manuscript. AA-H supervised the peritoneal dialysis procedure, provided the collection of all the human material and shared in the study design. MA-S was responsible for processing and interpreting the biopsy specimens.

Acknowledgements: this study was supported by the King Fahd Hospital Trust Fund and Dammam University Scientific Project Grant. The author extends his regards to Professor Abdul-Aziz Al-Quorain, Professor and Consultant of Internal Medicine and Gastroenterology, King Fahd University Hospital for his help. The author also thanks Dr. Abdullah AlHwiesh, Associate Professor of Internal Medicine and Nephrology as well as Professor Mohammad Al-Shawarby, Professor of Pathology; Dammam University for all the help and support they offered during preparation of this research. In addition, the author appreciates all the help offered by the hemodiaysis and peritoneal dialysis staff.

Conflict of interests: the author declares no potential conflict of interests.

This work is licensed under a Creative Commons Attribution NonCommercial 3.0 License (CC BY-NC 3.0).

CCopyright I. Saeed Abdul-Rahman, 2013

Licensee PAGEPress, Italy

Italian Journal of Medicine 2013; 7:118-123

doi:10.4081/itjm.2013.118

\begin{abstract}
The prevalence of gastroesophageal reflux disease (GERD) in end-stage renal disease (ESRD) patients who are on regular hemodialysis (HD) and those who are on peritoneal dialysis (PD) has been a subject of discussion over the past decades. Although, to our knowledge, few studies have compared the prevalence of GERD in both groups of patients, yet most authorities believe that the disease is more prevalent in PD patients. The aim of the current study is to clarify the relation between dialysis modalities and GERD. The study involved 68 ESRD patients who are on regular hemodialysis (group 1) and 71 age- and sex-matched ESRD patients on peritoneal dialysis (group 2). All patients were subjected to esophago-gastroduodenoscopy for proper evaluation. The prevalence of GERD and reflux esophagitis (RE) was similar in the two groups (45.6\% vs $47.9 .2 \%$ and $36.8 \%$ vs $39.4 \%$ respectively) ( $\mathrm{P}>0.05)$. The duration of dialysis, serum creatinine of 7 or over, the presence of diabetes mellitus, body mass index of $27 \mathrm{~kg} / \mathrm{m}^{2}$ or over, smoking and the absence of $H$. pylori infection were significantly associated with GERD and RE, while the dialysis modality, age, gender, use of nonsteroidal anti-inflammatory drugs, hypoalbuminemia and aspirin use were not. This study shows a similar prevalence of GERD and RE in both HD and PD patients. The duration rather than the modality of dialysis correlated significantly with GERD and RE.
\end{abstract}

\section{Introduction}

Gastroesophageal reflux disease (GERD) develops when acidic gastric contents reflux into the esophagus. The condition is believed mainly to be due to an increase in the number of transient lower esophageal sphincter relaxation events. Other major mechanisms include decreased clearance of esophageal contents and reflux owing to impaired peristalsis, decreased gastric emptying with resultant reflux into the esophagus, and increased gastric acid production with a resultant increase in the potency of the reflux. GERD is associated with an increased incidence of acid-related gastrointestinal disorders. ${ }^{1-3}$ The association between GERD and end-stage renal disease (ESRD) remains unclear. In previous reports ${ }^{4}$ the prevalence of GERD in ESRD was approximately $24 \%$ in the 418 stable hemodialysis (HD) patients who did not undergo endoscopic evalu- 
ation. Compared to the reported prevalence of GERD in 6010 Japanese adults (16.3\%), there was a higher prevalence of GERD in ESRD patients who underwent $\mathrm{HD}(24.2 \%){ }^{5}$ Peritoneal dialysis (PD) on the other hand has been accused of being associated with higher prevalence of GERD than HD. One study ${ }^{6}$ reported an incidence of $44.7 \%$ compared to $18.9 \%$ in HD patients. This concept was supported by other studies ${ }^{7-9}$ that reported an incidence of GERD ranging between $25.8 \%$ and $37.7 \%$ in PD patients. These studies, however, were based on a questionnaire that evaluated patients' symptoms suggesting the diagnosis of GERD, and they were not supported by endoscopic evaluation. In addition, there have been conflicting data regarding the prevalence of GERD and gastroesophageal mucosal lesions in patients on PD. In an attempt to resolve this issue, we have conducted a prospective and observational study to identify the prevalence of GERD and esophageal mucosal lesions (as proved by endoscopic examination) in the two groups of patients.

\section{Materials and Methods}

This study was conducted in the period between January $1^{\text {st }} 2008$, and February $1^{\text {st }} 2012$, at the King Fahd University Hospital, Al-Khobar, Saudi Arabia, and was carried out according to the Declaration of Helsinki. After obtaining written informed consent, the 139 ESRD patients with dyspeptic symptoms were included in this prospective study. Patients were divided into two groups: group 1 consisted of 68 patients on HD: 37 (54.4\%) males and 31 (45.6\%) females. Group 2 consisted of 71 patients on PD: 37 (52.1\%) males and 34 (47.9\%) females ( $\mathrm{P}>0.05)$. Fifty-nine patients $(86.8 \%)$ in group 1 and $65(91.5 \%)$ in group 2 were Saudis. Mean age was $47.5 \pm 14.3$ years and $45.9 \pm 12.5$ in groups 1 and 2 , respectively $(\mathrm{P}>0.05)$. Among the 71 patients in group 2, $51(71.8 \%)$ were on continuous ambulatory peritoneal dialysis, and 20 $(28.2 \%)$ on automated peritoneal dialysis. Patients with previous gastric surgery, and those who had received proton pump inhibitors or drugs with potential gastrointestinal motility effects, within the last three months were excluded from the study. Information concerning duration and type of dialysis and predialytic renal functions and other blood tests were obtained from the patients' charts (Table 1). All patients were subjected to upper gastrointestinal endoscopy with an Olympus GIF Q 230 videofiberscope; gastric biopsies were obtained and examined histologically for evidence of $H$. pylori. Gastric biopsy specimens were obtained from ulcer edge, erosion sites, body and antrum. The formalin-fixed, paraffin-embedded tissue sections were stained with hematoxylin and eosin, as well as modified Giemsa stain to detect $H$. pylori. All biopsy specimens were interpreted by one histopathologist. Patients were considered to be infected with $H$. pylori if the histological examination was positive. The statistical analysis was performed using SPSS for Windows version 5.0 (SPSS Inc. Chicago, IL, USA). Numerical values were given as mean $\pm \mathrm{SD}$, and t-test was used when appropriate. The $\chi^{2}$ test was used for nominal data. Fisher's exact test was applied. P values $<0.05$ were considered significant. Logistical regression analysis was carried out to evaluate the independent influence of each variable to GERD and reflux esophagitis (RE).

Table 1. Patients' characteristics.

\begin{tabular}{lccc}
\hline & HD (no. $\%)$ & PD (no. $\%)$ & P \\
\hline Total & $68(48.9)$ & $71(51.1)$ & NS \\
\hline Female/male & $31 / 37(45.6 / 54.4)$ & $34 / 37(47.9 / 52.1)$ & NS \\
\hline Saudis & $59(86.8)$ & $65(91.5)$ & NS \\
\hline Age $($ mean \pm SD) & $47.5 \pm 14.3$ & $45.9 \pm 12.5$ & NS \\
\hline Mean duration of dialysis (months) & $37 \pm 12.2$ & $32 \pm 14.2$ & NS \\
\hline Smoking $\geq 10$ years & $25(36.8)$ & $27(38.1)$ & NS \\
\hline DM & $38(55.9)$ & $42(59.2)$ & NS \\
\hline BMI $\geq 27$ & $15(22.1)$ & $18(25.4)$ & NS \\
\hline BUN $(m g / d L)(m e a n)$ & $86 \pm 11.2$ & $83 \pm 10.8$ & NS \\
\hline Creatinine $(\mathrm{mg} / \mathrm{dL})($ mean) & $11 \pm 2.8$ & $10 \pm 3.3$ & NS \\
\hline Albumin $\geq 3 \mathrm{~g} / \mathrm{dL}$ & $35(51.5)$ & $32(45.1)$ & NS \\
\hline Hgb $\leq 10 \mathrm{~g} / \mathrm{dL}$ & $27(39.7)$ & $25(35.2)$ & $\mathrm{NS}$
\end{tabular}

HD, hemodialysis; PD, peritoneal dialysis; NS, not significant; SD, standard deviation; DM, diabetes mellitus; BMI, body mass index; BUN, blood urea nitrogen; creatinine, serum creatinine; Hgb, hemoglobin. 


\section{Results}

Demographic characteristics of the patients are shown in Table 1. The biochemical parameters include: blood urea nitrogen, serum creatinine, fasting blood sugar and serum albumin. The mean age and male/female ratio were the same in both groups $(\mathrm{P}>0.05)$. The mean duration of dialysis was $37 \pm 12.2$ months in group 1 and $32 \pm 14.3$ months in group $2(\mathrm{P}>0.05)$. Diabetes mellitus was diagnosed in $55.9 \%$ of $\mathrm{HD}$ and $59.2 \%$ of $\mathrm{PD}$ dialysis patients $(\mathrm{P}>0.05)$. Body mass index (BMI) of $27 \mathrm{~kg} / \mathrm{m}^{2}$ or over was seen in $15(22.1 \%) \mathrm{HD}$ patients and in 18 (25.4\%) PD patients ( $\mathrm{P}>0.05)$. Twenty-five (36.8\%) HD and 27 (38.1\%) PD patients had been smokers for more than ten years $(\mathrm{P}>0.05)$. The prevalence of the different upper gastrointestinal tract lesions in all patients is shown in Table 2. The prevalence of GERD at the end of the study was similar in the two groups ( $45.6 \%$ vs $47.9 \%, \mathrm{P}>0.05)$. In addition, there was no statistically significant difference in the prevalence of RE between HD and PD patients (36.8\% vs $39.4 \%, P>0.05$ ) (Figures 1 and 2). Patients with GERD and $\mathrm{RE}$ had a longer duration of dialysis than those without (13.1 \pm 9.6 vs $7.8 \pm 6.2$ months) in group 1 $(\mathrm{P}<0.05)$, and $13.5 \pm 6.9$ vs $7.1 \pm 5.8$ months in group 2 $(\mathrm{P}<0.05)$ (Figures 1 and 2). Multivariate logistical regression analysis in groups 1 and 2 showed that duration of dialysis (12 months or more) [odds ratio (OR) 4.7, 95\% confidence interval (CI): 1.5-13.2], high serum creatinine $(\geq 7.0 \mathrm{mg} / \mathrm{dL})$ (OR 6.78, 95\% CI: 1.12 45.82), BMI $27 \mathrm{~kg} / \mathrm{m}^{2}$ or over (OR 5.78, 95\% CI: 1.01 32.5), smoking (OR 4.9, 95\% CI: 1.3-16.9), diabetes mellitus (OR 5.4, 95\% CI: 1.4-15.2) and absence of $H$. pylori infection (OR 3.58, 95\% CI: 1.11-16.8) but not dialysis modality, age, gender, use of nonsteroidal antiinflammatory drugs (NSAIDs), aspirin or hypoalbuminemia (defined as serum albumin $\leq 3 \mathrm{gm} / \mathrm{dL}$ ) were significantly associated with GERD and RE. In addition, there was a greater prevalence of antral gastritis and duodenal ulcers in $H$. pylori negative than in $H$. $p y$ lori positive patients in both groups: 9 of $1,(69.2 \%) \mathrm{vs}$ 4 of $13(30.8 \%)$ cases $(\mathrm{P}<0.01)$ and 6 of $8(75 \%)$ cases vs 2 of $8(25 \%)$ cases $(\mathrm{P}<0.05)$ in group $1 ; 11$ of 16 $(68.8 \%)$ cases vs 5 of $16(31.3 \%)$ cases $(\mathrm{P}<0.01)$ and 3 of $4(75 \%)$ cases $v s 1$ of $4(25 \%)$ cases $(\mathrm{P}<0.05)$ in group 2 (Table 2).

\section{Discussion}

Gastroesophageal reflux disease, a highly prevalent disorder, is defined as reflux of gastroduodenal content to the esophagus and includes RE and Barrett's esophagus. ${ }^{10,11}$ The symptoms of GERD typically include dyspepsia, pyrosis, pharyngeal, laryngeal and tracheal symptoms. ${ }^{12,13}$ The pathophysiology of GERD is multifactorial and depends on interaction between defense mechanisms and pathophysiological factors. The lower esophageal sphincter (LES) normally works in conjunction with the diaphragm to create a physical barrier against the reflux of gastric contents into the esophagus. ${ }^{14}$ Transient relaxation of LES may occur more often in patients with ESRD.${ }^{15}$ Esophageal motility disorders and delayed gastric emptying may also play a role in the development of GERD in ESRD. ${ }^{15}$

Studies in patients with ESRD showed a definite association between chronic renal failure (CRF) and delayed gastric emptying in peritoneal dialysis ${ }^{16}$ as well as in hemodialysis. ${ }^{17-19}$ Other studies linked the delayed gastric emptying to the hypoalbuminemic states observed in patients with ESRD. ${ }^{20,21}$ Prokinetics administered to hypoalbuminemic patients not only increased the gastric emptying, but also improved the plasma albumin levels. The situation however, is muddled by other controversial studies that have not demonstrated any decrease in gastric emptying in HD patients. ${ }^{22,23} \mathrm{Ko}$ et al..$^{24}$ demonstrated that uremic patients undergoing dialysis had gastric dysrhythmias that deteriorated after HD. The presence of an autonomic neuropathy may also confound the issue. Dumitrascu et al. ${ }^{25}$ demonstrated that patients with CRF had delayed gastric emptying if parasympathetic and sympathetic neuropathies are both present. Diabetics are at an increased risk of autonomic neuropathy, and they certainly constitute a large proportion of patients with ESRD. Delayed gastric

Table 2. The prevalence of upper gastrointestinal tract lesions in all patients.

\begin{tabular}{lcccccccc}
\hline GI lesion & No. (\%) & \multicolumn{2}{c}{ Group 1 } & P & No. (\%) & \multicolumn{2}{c}{ Group 2 } & P \\
& & HP- (no. \%) & HP+ (no. \%) & & & HP- (no. \%) & HP+ (no. \%) & \\
\hline GERD+RE & $31(45.6)$ & $23(74.2)$ & $8(25.8)$ & $<0.01$ & $34(47.9)$ & $22(64.7)$ & $12(35.3)$ & $<0.01$ \\
\hline Antral gastritis & $13(19.1)$ & $9(69.2)$ & $4(30.8)$ & $<0.01$ & $16(22.5)$ & $11(68.8)$ & $5(31.3)$ & $<0.01$ \\
\hline Erosive gastritis & $10(14.7)$ & $5(50)$ & $5(50)$ & NS & $13(18.3)$ & $7(53.8)$ & $6(46.2)$ & NS \\
\hline Gastroduodenitis & $6(8.8)$ & $4(66.7)$ & $2(33.3)$ & $<0.05$ & $4(5.6)$ & $2(50)$ & $2(50)$ & NS \\
\hline Duodenal ulcer & $8(11.8)$ & $6(75)$ & $2(25)$ & $<0.05$ & $4(5.6)$ & $3(75)$ & $1(25)$ & $<0.05$ \\
\hline
\end{tabular}

GI, gastrointestinal; HP-, H. pylori negative; HP+, H. pylori positive; GERD, gastroesophageal reflux disease; RE, reflux esophagitis; NS, not significant. 


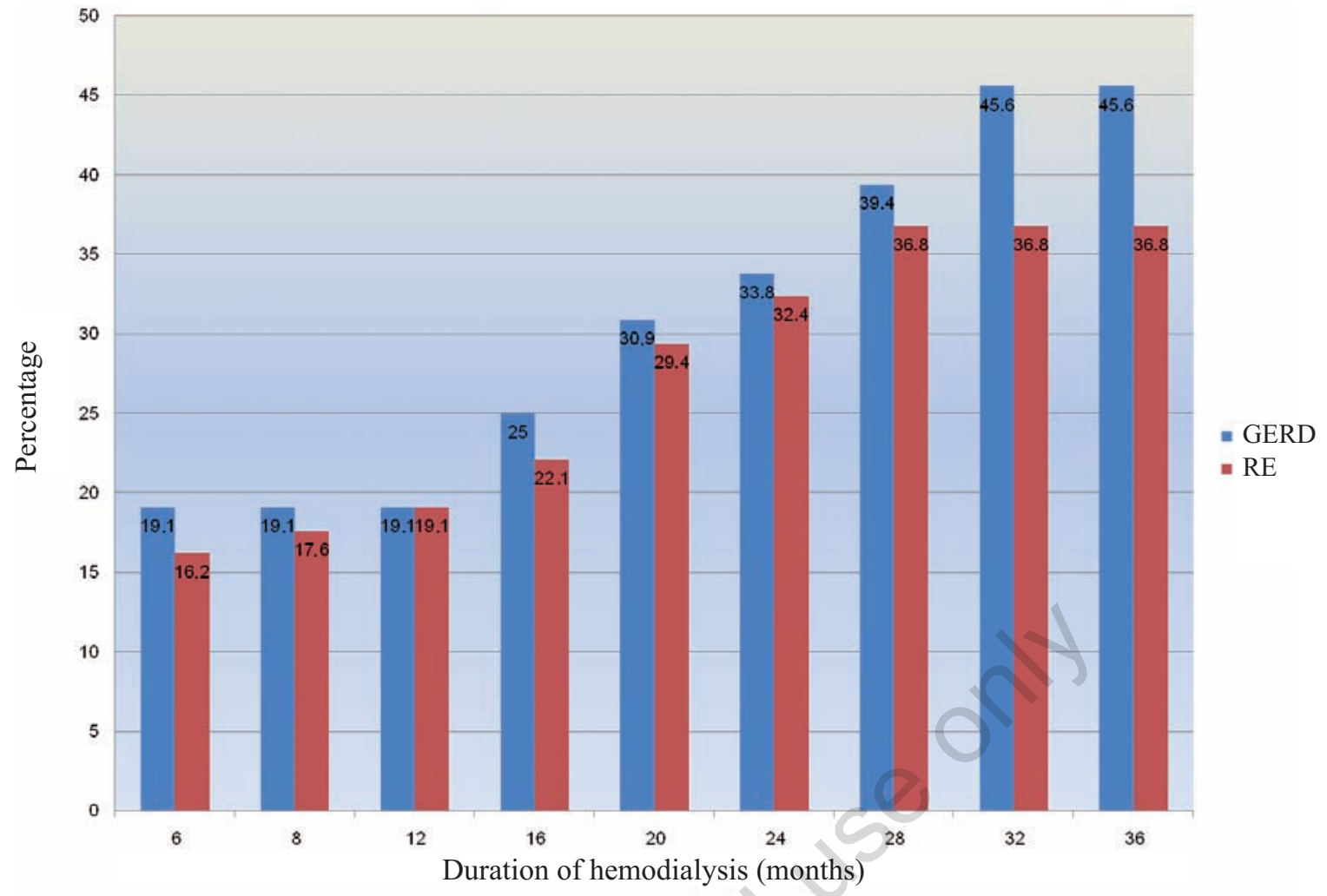

Figure 1. Prevalence of gastroesophageal reflux disease (GERD) and reflux esophagitis (RE) in relation to duration of hemodialysis.

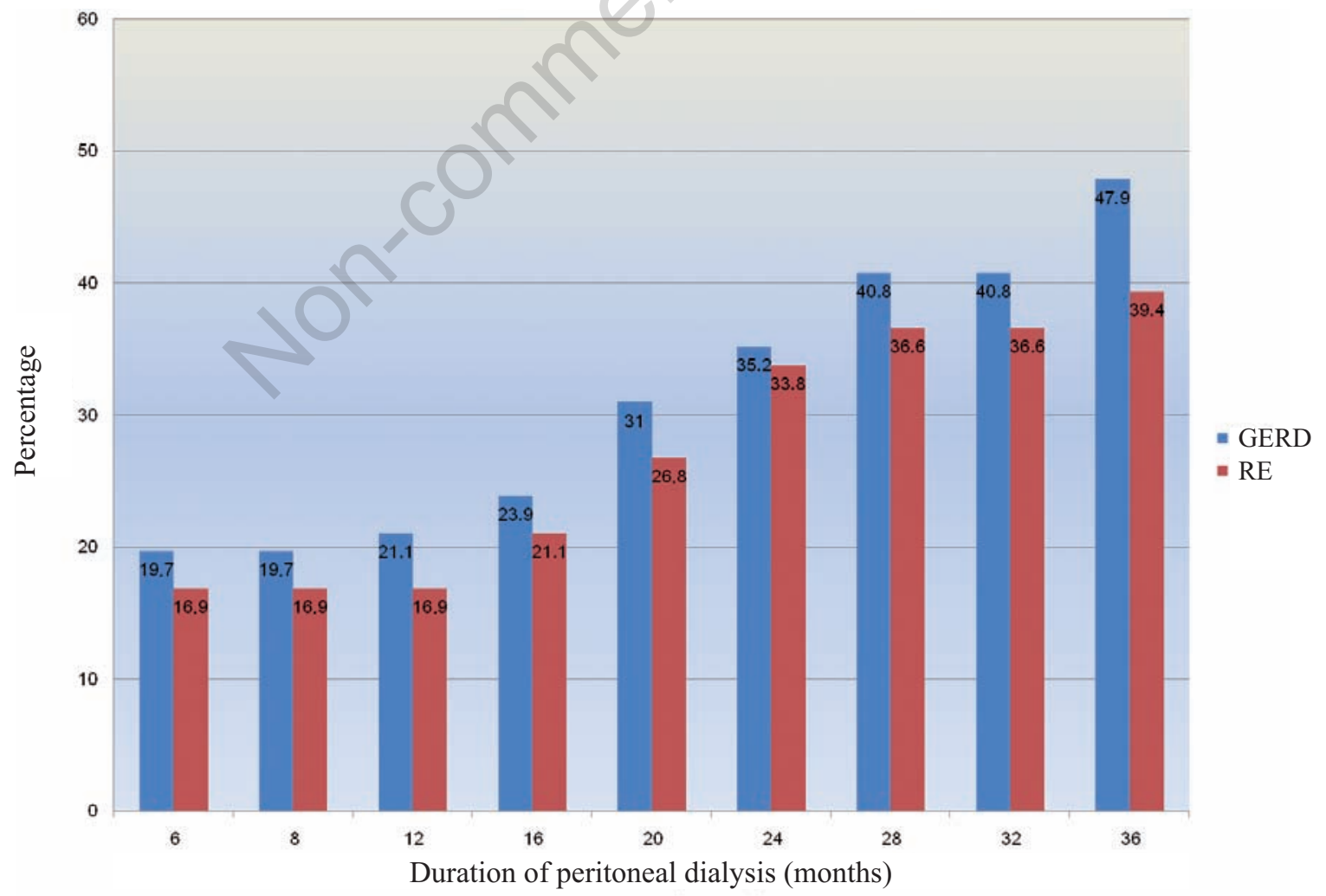

Figure 2. Prevalence of gastroesophageal reflux disease (GERD) and reflux esophagitis (RE) in relation to duration of peritoneal dialysis. 
emptying and autonomic neuropathy in HD as well as in PD patients are, therefore, the leading factors in the pathogenesis of GERD in ESRD.

Findings have been inconsistent regarding the relationship between gastric acid secretion and uremia; however, most authors believe that chronic renal failure is associated with hyperchlorhydria. ${ }^{26-29}$ Moreover, individuals with impaired renal function have elevated serum gastrin level due to reduced excretion and impaired metabolism in the liver degradation of gastrin. ${ }^{26-}$ ${ }^{28}$ Straathof et al. ${ }^{30}$ reported that post-prandial plasma concentrations of gastrin, in addition to its effect on gastric acid secretion, reduce lower esophageal sphincter pressure and increase the transient lower esophageal sphincter relaxations associated with reflux. These factors collectively may explain the higher prevalence of GERD in our group of patients with ESRD.

In a recent publication, Strid and his colleagues ${ }^{7}$ found delayed gastric emptying in $36 \%$ of their patients with ESRD, with a higher prevalence in peritoneal dialysis patients compared to chronic renal failure patients who are not on dialysis. They explained that this was due to the increased intra-abdominal pressure induced by the intraperitoneal dialysis fluid, along with other factors. Instead, based on our clinical observation, we found that GERD is linked to the uremic state. Our assumption is supported by another study that recruited 61 PD patients and showed a strong correlation between intraperitoneal pressure and intraperitoneal volume, but failed to show any influence of the intraperitoneal pressure on the occurrence of GERD in those patients. ${ }^{31} \mathrm{We}$ accept that it is plausible that peritoneal dialysis fluids increase the intra-abdominal pressure in PD, but we did not find any statistically significant differences in the prevalence of GERD between HD and PD patients and this throws doubts on the traditional accusation of increased intra-abdominal pressure on GERD in PD patients (Table 1). Pregnancy, for example, is a significant risk factor for GERD; as many as $66 \%$ of females have complained of GERD-type symptoms during the later stages of pregnancy. ${ }^{32,33}$ GERD during pregnancy has frequently been attributed to increased intra-abdominal pressure. This assumption, however, was denied in a novel study in which tense ascites was used as a model of pseudopregnancy. ${ }^{34}$ Patients in the study did not suffer a higher incidence of reflux, despite high intraabdominal pressure. Hence, researchers believe that the hormonal changes of pregnancy (rather than the high intra-abdominal pressure) directly decrease the lower esophageal sphincter pressure. Another example of increased intra-abdominal pressure is the patient with chronic liver disease and ascites. The cause and mechanism of GERD in those patients have not been clarified. However, it is strongly believed that more than one factor is involved. First, the changes in gastrointestinal hormones, namely plasma vasoactive peptide and neu- rotensin that reduce the lower esophageal sphincter pressure. ${ }^{35}$ Second, delayed gastric emptying that is influenced by the extent of liver damage. ${ }^{36}$ Third, the influence of esophageal varices on the lower esophageal sphincter function and on esophageal emptying. ${ }^{37,38}$ And fifth, the increased level of nitric oxide in those patients and its relation to the transient lower esophageal sphincter relaxation..$^{39}$ A recent multicenter study, ${ }^{40}$ involving 1280 patients with chronic liver disease did not show a significant correlation between increased intra-abdominal pressure due to ascites and the high incidence of GERD and RE in cirrhotic patients. The recent prospective study by Schechter et al. ${ }^{38}$ failed to demonstrate any correlation between increased intra-abdominal pressure due to ascites and GERD.

In our study, the duration of CRF, serum creatinine level, diabetes mellitus, smoking, body mass index 27 $\mathrm{kg} / \mathrm{m}^{2}$ or over and absence of $H$. pylori, were significantly associated with GERD and RE, while dialysis modality, age, gender, use of NSAIDs or aspirin and hypoalbuminemia were not.

Compared to HD, peritoneal dialysis is thought to provide a better preservation of residual renal function; an important factor that has been shown in a recent multivariate analysis study to protect $\mathrm{PD}$ patients from developing gastrointestinal symptoms. ${ }^{8}$ In a previous study, Anderson et al. ${ }^{6}$ reported that age under 60 years and body mass index $27 \mathrm{~kg} / \mathrm{m}^{2}$ or over, but not diabetes mellitus, were predictors of GERD in dialysis patients, while Dong et al. ${ }^{8}$ reported that neither age nor diabetes mellitus were predictors of gastrointestinal symptoms in PD patients. Our study, on the contrary, shows that diabetes mellitus but not age is a predictor of GERD in both HD and PD patients.

\section{Conclusions}

The present study demonstrates a similar prevalence of GERD and RE in both PD and HD patients, a positive correlation between the duration of dialysis, severity of CRF, diabetes mellitus, smoking, body mass index $27 \mathrm{~kg} / \mathrm{m}^{2}$ or over, absence of $H$. pylori infection and GERD or RE. On the other hand, there was a negative correlation between dialysis modalities, age, gender, use of NSAIDs, hypoalbuminemia and GERD, which strongly suggests that PD per se should not be accused of being responsible for gastroesophageal disease. Further evaluation in a larger population of HD and PD patients with a control group is needed.

\section{References}

1. Milito G, Taccone-Gallucci M, Brancaleone C, et al. Assessment of the upper gastrointestinal tract in hemodialysis patients awaiting renal transplantation. Am J Gastreneterol 1983;78:328-31. 
2. Milito G, Taccone-Gallucci M, Brancaleone C, et al. The gastrointestinal tract in uremic patients on long-term hemodialysis. Kidney Int 1985;17:157-60.

3. Ala-Kaila K. Upper gastrointestinal findings in chronic renal failure. Scand J Gastrointerol 1987;22:37276.

4. Kawaguchi Y, Mine T, Kawana I, et al. Gastroesophageal reflux disease in hemodialysis patients. Tokai J Exp Med 2009;34:48-52.

5. Furukawa N, Iwakiri R, Koyama T, et al. Proportion of reflux esophagitis in 6010 Japanese adults: prospective evaluation by endoscope. J Gastroenterol 1999 34:441-4.

6. Anderson JE, Yim KB, Crowell. Prevalence of gastroesophageal reflux disease in peritoneal dialysis and hemodialysis patients. Adv Perit Dial 1999;15:75-8.

7. Strid H, Fjell A, Simren M, Bjornsson ES. Impact of dialysis on gastroesophageal reflux, dyspepsia, and proton pump inhibitor treatment in patients with chronic renal failure. Eur J Gastroenterol Hepatol 2009;21:137-42.

8. Dong R, Guo ZY. Gastrointestinal symptoms in patients undergoing peritoneal dialysis: multivariate analysis of correlated factors. World J Gastroenterol 2010;16:2812-7.

9. Cano AE, Neil AK, Kang JY, et al. Gastrointestinal symptoms in patients with end-stage renal disease undergoing treatment by hemodialysis or peritoneal dialysis. Am J Gastroenterol 2007;102:1990-7.

10. Guozoing P, Guoming X, Hulping G, et al. Beijing and Shanghai to investigate the epidemiology of gastroesophageal reflux symptoms. Zhang Hua Xiao Hua Za Zhi 1999;19:223-6.

11. Gisbert JP, Cooper A, Karagiannis D, et al. Impact of gastroesophageal reflux esophagitis on patients' daily lives: a European observational study in the primary care setting. Health Qual Life Outcomes 2009;7:60.

12. Fock KM, Talley NJ, Fass R, et al. Asia-Pacific consensus on the management of gastroesophageal reflux esophagitis: update. J Gastroenterol Hepatol 2008; 23:504.

13. Hammer HF. Reflux-associated laryngitis and laryngopharyngeal reflux: a gastroenterologist point of view. Dig Dis Sci 2009;27:14-7.

14. Floch MH, Lacy BE. Esophageal reflux and motility update: GERD, NERD and spasm. J Clin Gastroenterol 2008;42:557.

15. Fallone CA, Mayrand S. Gastroesophageal reflux and hyperacidity in chronic renal failure. Perit Dial Int 2001;21:S295-9.

16. Brown-Cartwright D, Smith HJ, Feldman M. Gastric emptying of an indigestible solid in patients with end stage renal disease on continuous ambulatory peritoneal dialysis. Gastroenterology 1998;95:49-51.

17. Kao CH, Hsu YH, Wang SJ. Delayed gastric emptying in patients with chronic renal failure. Nucl Med Commun 1996;17:164-7.

18. Ravelli AM, Lederman SE, Bisset WM, et al. Foregut motor function in chronic renal failure. Arch Dis Child 1992;67:1343-7.

19. McNamee PT, Moore GW, McGeown MG, Doherty CC. Gastric emptying in chronic renal failure. Br Med J (Clin Res Ed) 1985;291:310-1.

20. Ross EA, Koo LC. Improved nutrition after the detection and treatment of occult gastroparesis in non-diabetic dialysis patients. Am J Kidney Dis 1998;31:62-6.

21. Silang R, Regalado M, Cheng TH, Wesson DE. Proki- netic agents increase plasma albumin in hypoalbuminemic chronic dialysis patients with delayed gastric emptying. Am J Kidney Dis 2001;37:287-93.

22. Wright RA, Clemente T, Wathen R. Gastric emptying in patients with chronic renal failure receiving hemodialysis. Arch Intern Med 1984;144:495-6.

23. Soffer EE, Geva B, Helman C, et al. Gastric emptying in chronic renal failure patients on hemodialysis. J Clin Gastroenterol 1987;9:651-3.

24. Ko CW, Chang CS, Wu MJ, Chen CH. Gastric Dysrhythmia in uremic patients on maintenance hemodialysis. Scand J Gastroenterol 1998;33:1047-51.

25. Dumitrascu DL, Barnert J, Kirschner T, Wienbeck M. Antral emptying of semisolid meal measured by realtime ultrasonography in chronic renal failure. Dig Dis Sci 1995:636-44.

26. Korman MG, Laver MC, Hansky J. Hypergastrinemia in chronic renal failure. Br Med J 1972;1:109-10.

27. Hallgren R, Karlsson FA, Lundqvist G. Serum levels of immunoreactive gastrin: influence of kidney function. Gut 1978;19:207-13.

28. Carlei F, Caruso U, Lezoche E, et al. Hyperplasia of antral G cells in uremic patients. Digestion 1984;29:26-30.

29. Kang JY. The gastrointestinal tract in uremia. Dig Dis Sci 1993;38:257-68.

30. Straathof JW, Lamers CB, Masclee AA. Effect of gastrin-17 on lower esophageal sphincter characteristics in man. Dig Dis Sci 1997;42:2547-51.

31. Dejardin A, Robert A, Coffin E. Intraperitoneal pressure in PD patients: relationship to intraperitoneal volume, body size and PD-related complications. Nephrol Dial Transplant 2007;22:1437-44.

32. Bor S, Kitapcioglu G, Dettmar P, Baxter T. Association of heartburn during pregnancy with the risk of gastroesophageal reflux disease. Clin Gastroenterol Hepatol 2007;5:1035-9.

33. Richter JE. Gastroesophageal reflux disease during pregnancy. Gastroenterol Clin North Am 2003;32:235-8.

34. Van Thiel DH, Wald A. Evidence refuting a role for increasing abdominal pressure in the pathogenesis of heartburn associated with pregnancy. Am J Obstet Gynecol 1981;140:420-24.

35. Grassi M, Albaini B, De Matteis A, et al. Prevalence of dyspepsia in liver cirrhosis: a clinical and epidemiological investigation. Minerva Med 2001;92:7-12.

36. Ishizu H, Shiomi S, Kawamura E, et al. Gastric emptying in patients with chronic liver disease. Ann Nucl Med 2002;16:177-82.

37. Zhang J, Cui PL, Lv D, et al. Gastroesophageal reflux in cirrhotic patients without esophageal varices. World J Gastroenterol 2011;17:1753-8.

38. Schechter RB, Lemme EM, Coelho HS. Gastroesophageal reflux in cirrhotic patients with esophageal varices without endoscopic treatment. Arq Gastroenterol 2007;44:145-50

39. Magomedéminova AS, Esedov EM. Gastric juice levels of nitric oxide in relation to gastric acidicity in patients with upper digestive tract diseases. Klin Lab Diagn 2010;7:48-50.

40. Li B, Zhang B, Ma JW, et al. High prevalence of reflux esophagitis among upper endoscopies in Chinese patients with chronic liver disease. BMC Gastroenterol 2010;10:54-9. 